EPJ Web of Conferences 111, 11006 (2016)

DOI: $10.1051 /$ epjconf/201611111006

(C) Owned by the authors, published by EDP Sciences, 2016

\title{
Toward Reanalysis of the Tight-Pitch HCLWR-PROTEUS Phase II Experiments
}

\author{
Grégory Perret ${ }^{1, a}$, Efstathios Vlassopoulos ${ }^{1, b}$, Mathieu Hursin ${ }^{1,2}$, and Andreas Pautz ${ }^{1,2}$ \\ ${ }^{1}$ Paul Scherrer Institute, 5232 Villigen, Switzerland \\ ${ }^{2}$ Ecole Polytechnique Fédérale de Lausanne, 1015 Lausanne, Switzerland
}

\begin{abstract}
The HCLWR-Proteus Phase II experiments were conducted from 1985 to 1990 in the zero-power reactor Proteus at PSI in Switzerland. The experimental program was dedicated to the physics of high conversion light water reactors and in particular to the measurement of reactor parameters such as reaction rate traverses, spectral indices, absorber reactivity worths and void coefficients. The HCLWR experiments are especially interesting because they generated knowledge in the epithermal range of the neutron flux spectrum, for which little integral experimental data is available.

In an effort to assess the interest of this experimental data to validate modern nuclear data and improve their uncertainties, a preliminary re-analysis of selected configurations was conducted with Monte-Carlo codes (MCNP6/SERPENT2) and modern nuclear data libraries (ENDF/B-VII.0, JEFF-3.1.1 and JENDL-4.0). The spectral indices, flux spectra and sensitivity coefficients on $\mathrm{k}_{\infty}$ were calculated using cell models representative of the tight-pitch measurement configurations containing $11 \% \mathrm{PuO}_{2}-\mathrm{UO}_{2}$ fuel rods in different moderation conditions (air, water and dowtherm).

Spectral index predictions using the three nuclear data libraries agreed within two standard deviations with the measured values. The only exception is the Pu-242-captureto-Pu-239-fission ratio, which was overestimated with all libraries by more than four standard deviations, i.e. $13 \%$, in the non-moderated configuration. In this configuration, $\mathrm{Pu}-242$ captures are few since the flux spectrum in the $\mathrm{Pu}-242$ capture resonance region (between $1 \mathrm{eV}$ and $1 \mathrm{keV}$ ) is small making this spectral index hard to measure. Sensitivity coefficient predictions with both MCNP6 and SERPENT2 were in good agreement.
\end{abstract}

\section{Introduction}

New integral experiments dedicated to neutronic codes and data validation are scarce nowadays. This is due in part to the already enormous knowledge gathered along the years but also to the increasing costs and long-time commitment necessary to perform experiments. There are, however, at least two areas in which zero-power reactor neutronics experiments are still of value for modern codes and nuclear data libraries, namely for validation of reactor concepts different from the traditional Light Water Reactors and for more detailed investigations (e.g. within pin power distribution). The

\footnotetext{
a e-mail: gregory.perret@psi.ch

${ }^{b}$ Now at NAGRA, 5430 Wettingen, Switzerland
} 
first trend is illustrated by the investigation of the GEN-IV reactor concepts; the second by the development of new set of high-fidelity codes, like in the framework of CASL, whose aim is to provide a framework for improved multi-physics (i.e. coupling neutronics, materials and thermal-hydraulic phenomena) reactor analysis and which require detailed measurements for its validation.

Ironically enough, several experiments performed in the distant past (read 1960s-90s for neutronics) were performed on reactor concept not much different than those considered in GeN-IV or studied for the transition from the Light Water Reactors technology to fast reactors. A striking example is the Proteus experimental program, which was from the 1970s to 2000s dedicated in turn to Gas-Cooled Fast Reactors, High Conversion Light Water Reactors and High Temperature Reactors [1]. A cost effective solution, for both research institutes and international organisation, is to recycle these experiments by modeling them, in so-far as the experimental details are still available, with the codes and libraries of the day and validate them. We started this endeavour for the GCFR-Proteus program at Wonder-2012 [2], which will result in safeguarding these data in the IRPhE database [3].

In this work, we report on our initial effort to use the HCLWR-Proteus data for validation purposes. Section 2 summarizes the HCLWR-Proteus experiments and the selected configurations. Section 3 describes the code and pin-cell models used in this work and illustrate their characteristics with flux spectra and sensitivity coefficients and profiles. Section 4 presents spectral index predictions and their comparisons with the measurement performed during the HCLWR-Proteus experiments.

\section{Experiments}

The HCLWR-Proteus Phase II experiments were conducted from 1985 to 1990 in the Proteus reactor at Paul Scherrer Institute (PSI) in Switzerland. Proteus is a zero-power reactor featuring a cylindrical central cavity that is driven critical by a surrounding graphite region fueled with $5 \mathrm{w} \% \mathrm{UO}_{2}$ fuel pins. In the HCLWR-Proteus experiments, the central cavity was loaded with a $\mathrm{D}_{2} \mathrm{O}$ zone fueled with $5 \mathrm{w} \%$ $\mathrm{UO}_{2}$ fuel pins, a buffer region containing metallic natural uranium rods in air and a central test zone filled with a HCLWR-representative fuel lattice.

The experimental program was dedicated to the physics of high conversion light water reactors and in particular to the measurement of reactor parameters such as reaction rate traverses, spectral indices, absorber reactivity worths and void coefficients. In this paper we focus on the interpretation of the spectral index measurements performed in cores 7, 8 and 9. In cores 7,8 and 9, the test zone comprises a tight-pitch hexagonal lattice of fuel pins (pitch of $1.07 \mathrm{~cm}$; moderator-to-fuel ratio of 0.48; pitch-to-diameter of 1.12) loaded in light water (atmospheric conditions), air and dowtherm ${ }^{1}$, respectively. The fuel is $\mathrm{PuO}_{2}-\mathrm{UO}_{2}$ with about $11 \% \mathrm{PuO}_{2}$ of which $72 \%$ is fissile. This set of core configurations is highly interesting for validation purposes as it features spectral conditions in both the epithermal and fast ranges (see section 3.1 and 3.2) - for which experimental data are scarce.

Reaction rate ratios were measured using fission chambers and foils. Fissions in U-235 (F5), $\mathrm{Pu}-239$ (F9) and U-238 (F8) were respectively measured using U/Al, Pu/Al and $\mathrm{U}_{\text {metal }}$ or $\mathrm{UO}_{2}$ foils in an instrumented pin at the core center. The absolute reaction rates were determined using intercalibrated foils located together with absolutely calibrated fission chambers in a cavity $25 \mathrm{~cm}$ above the core center. Captures in U-238 (C8) was determined using $\mathrm{UO}_{2}$ foils in the instrumented rod. Because of the difficulty to manufacture and handle large enough $\mathrm{Pu}-241$ deposits, fissions in $\mathrm{Pu}-241$ (F1) required using $\mathrm{Al}$ catcher foils wrapped around the $\mathrm{Pu}-241$ deposits. Measuring the activity of the Al-foils in the fission chamber and in the center of the core allowed determining the absolute rate of $\mathrm{Pu}-241$ fissions ${ }^{2}$. Finally, captures in Pu-242 (C2) were measured by counting the $84 \mathrm{keV}$

\footnotetext{
${ }^{1}$ Dowtherm is an organic liquid used to simulate water with lower density; its effective moderation is 0.28 as compared to 0.48 for light water.

${ }^{2}$ The same Al-foils were also measured without the $\mathrm{Pu}-241$ deposit to correct for their activation by other radiations.
} 

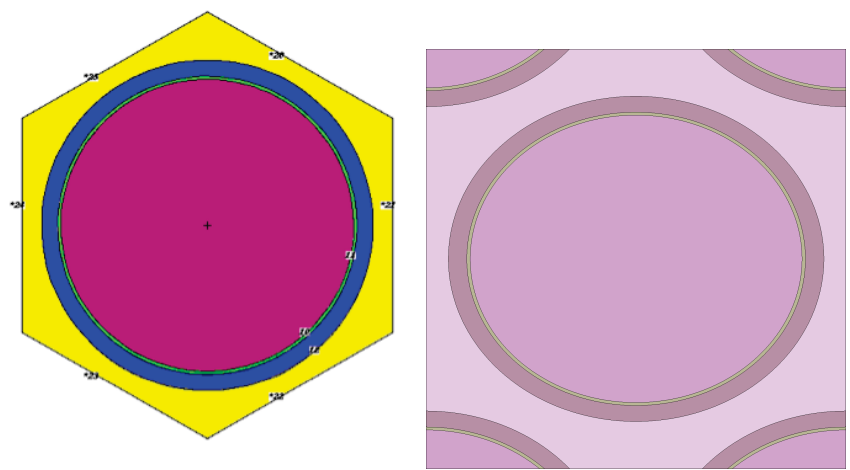

Figure 1. Pin-cell models - MCNP6 (left), Serpent (right).

$\gamma$-peak of Pu-243 emitted by a Pu-242 deposit irradiated in turn in a fission chamber located in the thermal column of the reactor and in the core center. The capture rate at the center of the core was determined using the relatively well known cross-section for capture in Pu-242 at the thermal energy ${ }^{3}$. Measurements of F1 and C2 were significantly harder than that of F5, F8, C8 and F9 and had higher uncertainties. Typical uncertainty on the measured reaction rate ratios are $1.5 \%-2 \%$ for $\mathrm{C} 8 / \mathrm{F} 9, \mathrm{~F} 8 / \mathrm{F} 9$ and $\mathrm{F} 5 / \mathrm{F} 9$, and $3 \%$ for F1/F9 and C2/F9 [4].

\section{Codes and Models}

In this work, we used the MCNP6 and SERPENT2 Monte-Carlo codes [5, 6] with the modern JEFF-3.1.1, ENDF/B-VII.0 and JENDL-4.0 nuclear data libraries [7-9]. The model presented here are pin cell models. Under the assumption that the center of the test zone of Proteus is representative of the neutronic conditions encountered in the infinite lattice, these models are sufficient to help characterize the experiments with flux spectrum and sensitivity profiles. In addition, they can be used to compare their predictions to the reaction rate ratio measured during the HCLWR-Proteus experiments, as the latter have been corrected for the low impact of the driver regions at the center of the core. Correction factors to account for leakage were calculated at the time of the experiments using diffusion theory and were less than $1 \%$ for the reaction rates measured in cores 7,8 and 9 , with the exception of C8/F9 in core $8(1.8 \%)$, confirming that the center of the Proteus test zone is representative of an HCLWR [4].

Fig. 1 shows radial cut-away views of the MCNP6 and SERPENT2 pin cell models. The reference fuel material compositions were corrected to account for the $\alpha$-decay of Pu-238 and $\beta^{-}$decay of Pu241 in U-234 and Am-241, respectively. The boundary conditions are reflective in the radial direction. For the flux and reaction rate calculations, the height of the cell is adjusted to reach criticality and performed k-eigenvalue calculations with about 100 million particles.

\subsection{Flux spectrum}

The flux and reaction rates are tallied on the fuel region spreading $\pm 2.5 \mathrm{~cm}$ around the median plan, where the spectrum is not significantly distorted by the axial leakage. For the flux tallying, we used

\footnotetext{
${ }^{3}$ Although the $\mathrm{Pu}-242$ thermal capture cross section should be well-known, the value taken from an outdated library may introduce some bias in $\mathrm{C} 2$ assessment.
} 


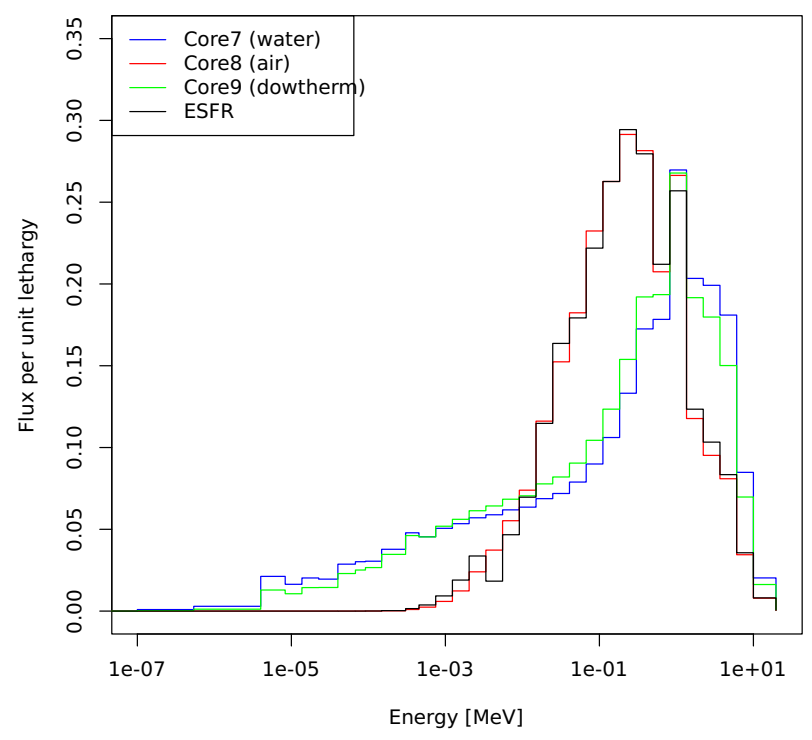

Figure 2. Flux spectra for the three studied configurations and the European sodium fast reactor.

the ECCO-33 energy group typically employed for fast reactor systems [10]. Typical uncertainty is lower than $0.1 \%$ for the bins in which the flux is not negligible.

Fig. 2 shows the flux spectra per unit lethargy for the three selected configurations and for the European sodium fast reactor. Calculations were performed with MCNP6 and ENDF/B-VII.0. The reference HCLWR core moderated with water at room temperature and pressure (core 7) has a softer spectrum than the sodium fast reactor, especially in the epithermal range from $10 \mathrm{eV}$ to $10 \mathrm{keV}$. The flux spectra of the voided HCLWR core (core 8) is similar to that of the sodium reactor - the main visible difference being the first sodium scattering resonance at $2.85 \mathrm{keV}$. Finally, the core moderated with dowtherm (core 9) is, as expected, in between the spectra of cores 7 and 8.

We calculated the same spectra with SERPENT2 and with JEFF-3.1.1 and JENDL-4.0 and obtained similar results. Fig. 3 shows, as an example, the flux spectra of the reference HCLWR core obtained with the three libraries. The maximum deviations are about $2 \%$, i.e. negligible. Differences in the spectral index predictions come therefore mainly from the tabulated cross section and not from the flux calculation.

\subsection{Sensitivity analysis}

Sensitivity coefficients and profiles are required to assess how representative an experiment, like HCLWR-Proteus, is of a design case, e.g. Reduced Moderation Boiling Water Reactors or fast reactors [11], and to reduce the a priori uncertainty on the prediction of the reactor parameter for the design case [12]. These quantities also provides additional physical insight on the modeled experiment. 

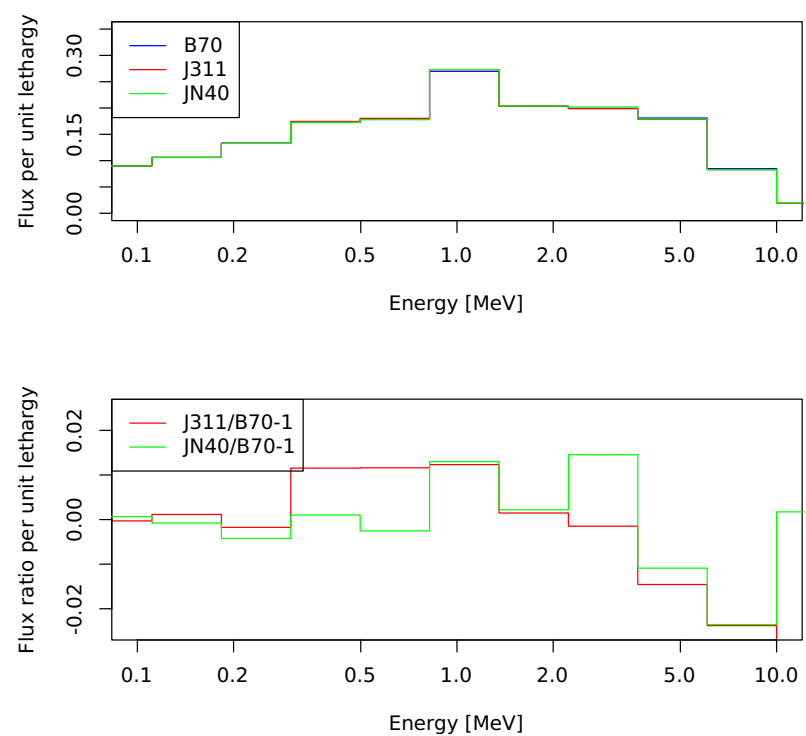

Figure 3. Flux spectra for different libraries (top) and differences (bottom).

Table 1. Sensitivity coefficients of $\mathrm{k}_{\infty}$ integrated on the whole spectra.

\begin{tabular}{cccc}
\hline Isotope & Reaction & Core 7 (Water) & Core 8 (Voided) \\
\hline & Total & $-0.146(1.5 \%)$ & $-0.337(1.1 \%)$ \\
U-238 & Inelastic & $-0.0379(1.9 \%)$ & $-0.128(0.9 \%)$ \\
& Capture & $-0.182(0.2 \%)$ & $-0.302(0.1 \%)$ \\
& Fission & $0.0639(0.4 \%)$ & $0.0937(0.4 \%)$ \\
\hline \multirow{4}{*}{ Pu-239 } & Total & $0.212(0.5 \%)$ & $0.388(0.3 \%)$ \\
& Inelastic & $-0.011(13 \%)$ & $-0.038(7 \%)$ \\
& Capture & $-0.155(0.2 \%)$ & $-0.045(1.2 \%)$ \\
& Fission & $0.368(0.3 \%)$ & $0.438(0.2 \%)$ \\
\hline
\end{tabular}

We calculated sensitivity coefficient relative to the infinite multiplication factor $\left(\mathrm{k}_{\infty}\right)$. In these calculations, the pin cell axial boundary conditions are reflective and the sensitivity coefficients are calculated on all regions of the model.

Tab 1 shows, for illustrative purposes, the dominant sensitivity coefficients for U-238 and Pu-239 reactions obtained with MCNP6 and JEFF-3.1.1 in the water-moderated and voided configurations and their relative $1 \sigma$ uncertainty. The coefficients show the total impact on $\mathrm{k}_{\infty}$, i.e. integrated over the whole energy domain, of the isotope-reaction pairs. We obtained the same sensitivity coefficients, i.e. within $2 \sigma$ for the non negligible isotope-reaction pairs, when using SERPENT2 and JEFF-3.1.1.

We can notice the enhanced importance of U-238 reactions - especially inelastic and capture reactions - in the voided configuration. Regarding $\mathrm{Pu}-239$ reactions, as expected, the impact of the 

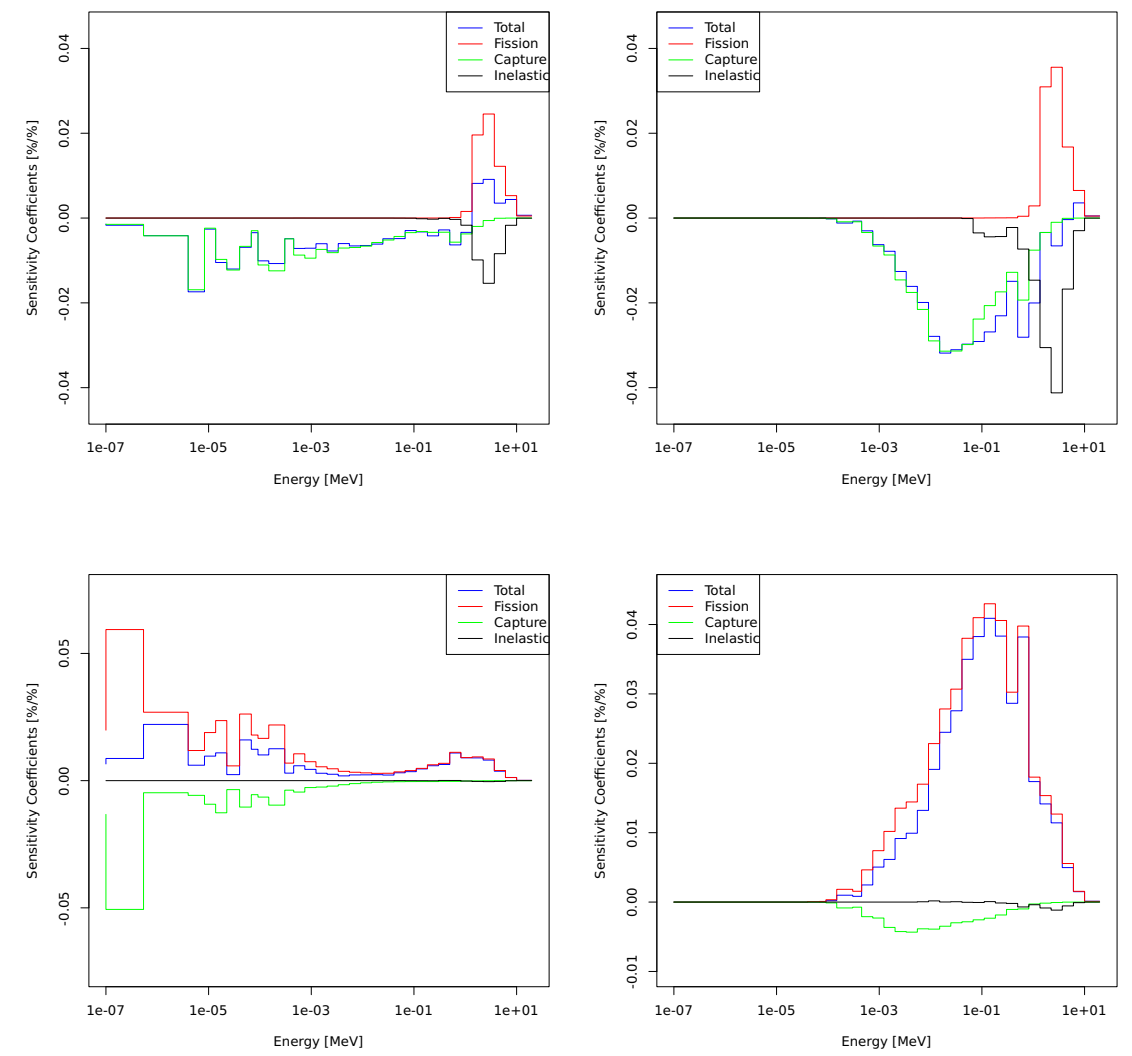

Figure 4. Sensitivity profiles for U-238 (top) and Pu-239 (bottom) in water-moderated (left) and voided (right) configurations.

capture cross section on $\mathrm{k}_{\infty}$ is strongly reduced in the voided configuration, and that of fission enhanced, pointing towards a better utilization of the $\mathrm{Pu}-239$ resources in fast spectra.

More insight can be gained by plotting the sensitivity profiles, i.e. sensitivity coefficient as a function of energy. Fig. 4 shows the sensitivity profiles for $\mathrm{k}_{\infty}$ calculated with MCNP6 and JEFF-3.1.1 in the water-moderated (core 7) and voided configurations (core 8). The main reactions, i.e. total, inelastic scattering, fission and capture reactions, are plotted for U-238 and Pu-239.

The importance of the inelastic scattering for U-238 in fast spectra is illustrated when comparing the two top plots, i.e. water-moderated configuration on the left and voided on the right. The impact of the inelastic scattering of $\mathrm{U}-238$ on $\mathrm{k}_{\infty}$ is largely enhanced above $1 \mathrm{MeV}$ and more than compensate the sensitivity coefficient for fission in U-238, rendering the total sensitivity coefficient positive above $2 \mathrm{MeV}$. The total sensitivity coefficient for U-238 is only positive above $10 \mathrm{MeV}$ in the moderated case. These graphs also shows the large compensating effects for U-238 reactions.

Finally, Pu-239 sensitivity profiles, illustrate, without surprise, the effect of the thermal fissions in the moderated case (left) as compared to the voided configuration (right). They also shows how the sensitivity of $\mathrm{k}_{\infty}$ to $\mathrm{Pu}-239$ reaction is only governed by fissions in fast spectra and the role of 
Table 2. Comparison of measured and calculated spectral indices.

\begin{tabular}{cccc}
\hline \multicolumn{4}{c}{ Core 7 (Water) } \\
\hline Index & B70 & J311 & JN40 \\
C8/F9 & $1.007 \pm 0.018$ & $1.007 \pm 0.018$ & $1.004 \pm 0.018$ \\
F8/F9 & $1.024 \pm 0.019$ & $1.005 \pm 0.019$ & $1.016 \pm 0.019$ \\
F5/F9 & $1.017 \pm 0.015$ & $1.016 \pm 0.015$ & $1.012 \pm 0.015$ \\
F1/F9 & $0.988 \pm 0.030$ & $0.988 \pm 0.030$ & $0.982 \pm 0.029$ \\
C2/F9 & $1.044 \pm 0.031$ & $1.020 \pm 0.030$ & $1.025 \pm 0.031$ \\
\hline \multicolumn{4}{c}{ Core 8 (Air) } \\
\hline Index & B70 & J311 \\
C8/F9 & $1.021 \pm 0.016$ & $1.018 \pm 0.016$ & $1.006 \pm 0.016$ \\
F8/F9 & $1.002 \pm 0.018$ & $0.983 \pm 0.018$ & $0.994 \pm 0.018$ \\
F5/F9 & $1.023 \pm 0.013$ & $1.025 \pm 0.013$ & $1.001 \pm 0.013$ \\
F1/F9 & $1.011 \pm 0.030$ & $1.022 \pm 0.031$ & $0.994 \pm 0.030$ \\
C2/F9 & $1.133 \pm 0.034$ & $1.230 \pm 0.037$ & $1.167 \pm 0.035$ \\
\hline \multicolumn{4}{c}{ Core $9($ Dowtherm) } \\
\hline Index & B70 & J311 \\
C8/F9 & $0.989 \pm 0.017$ & $0.992 \pm 0.017$ & $0.986 \pm 0.017$ \\
F8/F9 & $1.017 \pm 0.018$ & $0.999 \pm 0.018$ & $1.013 \pm 0.018$ \\
F5/F9 & $1.023 \pm 0.015$ & $1.024 \pm 0.015$ & $1.018 \pm 0.015$ \\
F1/F9 & $0.987 \pm 0.049$ & $0.990 \pm 0.050$ & $0.981 \pm 0.049$ \\
C2/F9 & $1.038 \pm 0.031$ & $1.035 \pm 0.031$ & $1.036 \pm 0.031$ \\
\hline
\end{tabular}

capture in moderated configuration - which impaired the effective burning of plutonium. We ran the same sensitivity profiles with SERPENT2 and JEFF-3.1.1 and found the same results (within $2 \sigma$ for the non negligible isotope-reaction pairs).

\section{Spectral Index Results}

Tab. 2 lists the calculated-to-measured ratio of the spectral indices detailed in section 2 and their $1 \sigma$ uncertainty. For the moderated cases (cores 7 and 9) all predictions agree with the measurements within $2 \sigma$. Slightly better agreement is obtained with JEFF-3.1.1. The spectral indices for the voided configuration (core 8 ) are also correctly predicted but for the $\mathrm{Pu}-242$ capture rate. The C2/F9 ratio is overpredicted by $13 \%$ to $23 \%$ depending on the library (i.e. by 4 to $6 \sigma$ ). The best predictions for the non moderated core are obtained, on average, with JENDL-4.0.

The difference in predictions for the $\mathrm{Pu}-242$ capture rate are directly related to the difference in the cross sections in the unresolved resonance and fast ranges (see Fig. 5 reproduced from [13]), in which the flux is non negligible (see Fig. 2). The source of the bias between predictions and the experimental value can be due to error in the measurement or post-processing of the measured value, or in the evaluations of the cross section.

Possible errors in the measurement stems from: the very low reaction rate due to the small $\mathrm{Pu}-$ 242 capture cross section above $1 \mathrm{keV}$ and the small amount of Pu-242 in the deposit $\left(0.2 \mathrm{mg} / \mathrm{cm}^{2}\right.$ on a $0.1-\mathrm{mm}$ thick aluminum foil), the difficulties to measure the low energy (84keV) gamma-ray lines $^{4}$ emitted by Pu-243 (which has a short half-life of 4.96 hours), and the calibration in the thermal

\footnotetext{
${ }^{4}$ due to self absorption and background of other radiations
} 


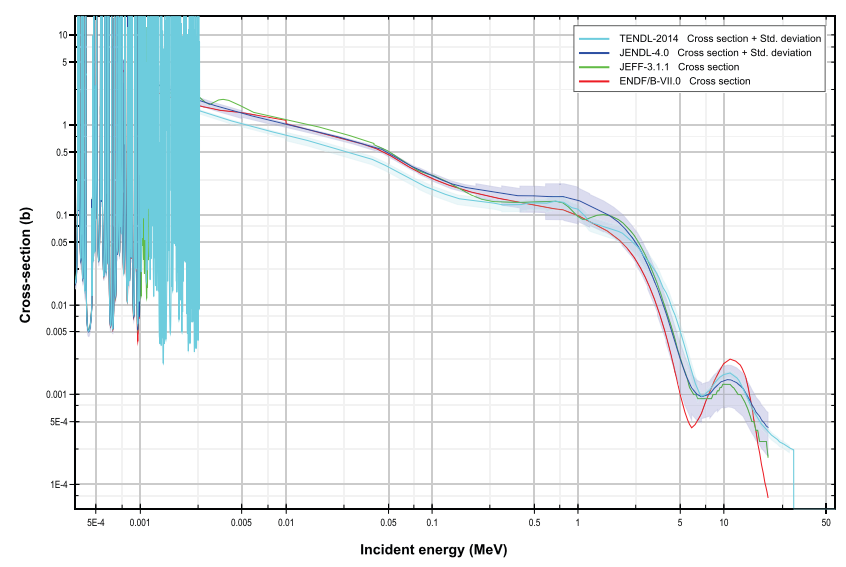

Figure 5. Pu-242 capture cross section above $1 \mathrm{keV}$ for different libraries.

column (see section 2), which uses the Pu-242 thermal cross-section of an outdated library. Further investigation of these sources of bias requires unfortunately a more detailed measurement report than the one currently available.

Regarding the nuclear data cross sections, Fig. 5 also shows that the uncertainty on the cross section, for example in JENDL-4.0 or TENDL-2014, is not negligible and that a bias may still exists today - as illustrated by the lower values of TENDL-2014 from $1 \mathrm{keV}$ to $1 \mathrm{MeV}$.

\section{Conclusion}

We presented a first re-analysis of the HCLWR-Proteus experiments with the Monte-Carlo codes MCNP6 and SERPENT2 and the modern nuclear data libraries ENDF/B-VII.0, JEFF-3.1.1 and JENDL-4.0. We focused on the tight pitch core configurations moderated with water or dowtherm and voided, and calculated the spectral indices measured in the center of the core.

The calculation models are simple pin cells, as they are representative of the spectral conditions encountered in the center of core in the selected HCLWR-Proteus core configurations. Results showed an excellent agreement (within 1-2 $\sigma$ ) for the C8/F9, F8/F9, F5/F9 and F1/F9 indices ${ }^{5}$ in the three core configurations. The $\mathrm{Pu}$-242-capture-to-Pu-239-fission index is well predicted only in the moderated configurations. In the voided configuration, in which the flux spectra is spanning from $1 \mathrm{keV}$ to $1 \mathrm{MeV}$, the index is over-predicted by $13 \%$ to $23 \%$ ( 4 to $6 \sigma$ ) depending on the library. In addition to possible biases in the evaluated cross sections of the selected libraries, errors in the measurements cannot be rejected. Errors could stems from the low measurement signal (few Pu-242 captures in the unresolved resonance range and high background) or the measurement method (comparison to thermal spectra measurement requiring the use of outdated $\mathrm{Pu}-242$ thermal cross sections), and will require further investigations.

Finally, we calculated sensitivity coefficients and profiles on $\mathrm{k}_{\infty}$ with MCNP6 and SERPENT2 and the JEFF-3.1.1 library. Results are consistent between the codes, illustrate the physics of the watermoderated and voided core configurations and will serve to determine if the experimental results could help design HCLWR or fast reactors.

\footnotetext{
${ }^{5} \mathrm{C}$ stands for capture, $\mathrm{F}$ for fission and 5, 8, 9 and 1 for $\mathrm{U}-235, \mathrm{U}-238, \mathrm{Pu}-239$ and $\mathrm{Pu}-241$, respectively.
} 


\section{References}

[1] G. Perret, http://www.psi.ch/erp/proteus (2015)

[2] G. Perret, K.A. Jordan, Tech. rep., Paul Scherrer Institut, Villigen, 5232, Switzerland (2012)

[3] K. Jordan, G. Perret, J. Bess, Benchmark Evaluation of PROTEUS Gas-Cooled Reactor Experiments (2014)

[4] H. Berger, R. Böhme, R. Chawla, H. Hager, O. Köberl, R. Seiler, T. Williams, Tech. rep., Paul Scherrer Institute, Villigen, 5232, Switzerland (1993)

[5] T. Goorley, Tech. rep., LA-UR-14-24680 - Los Alamos National Laboratory (2014)

[6] J. Leppänen, http://montecarlo.vtt.fi/index.htm (2015)

[7] A. Santamarina, D. Bernard, P. Blaise, M. Coste, A. Courcelle, T. Huynh, C. Jouanne, P. Leconte, O. Litaize, S. Mengelle et al., The JEFF-3.1.1 Nuclear Data Library - JEFF Report 22 (2009), www.nea.fr/html/dbdata/nds_jefreports/

[8] M. Chadwick, P. Obložinský, M. Herman, N. Greene, R. McKnight, D. Smith, P. Young, R. MacFarlane, G. Hale, S. Frankle et al., Nuclear Data Sheets 107, 2931 (2006), evaluated Nuclear Data File ENDF/B-VII.0

[9] K. Shibata, O. Iwamoto, T. Nakagawa, N. Iwamoto, Journal of Nuclear Science and Technology 48, 1 (2012)

[10] E. Sartori, Tech. rep., NEA DAta Bank, Gif-sur-Yvette, France (1990)

[11] T. Iwamura, S. Uchikawa, T. Okubo, T. Kugo, H. Akie, Y. Nakano, T. Nakatsuka, Nuclear Engineering and Design 236, 1599 (2006)

[12] O. Leray, C. Vaglio-Gaudard, J.P. Hudelot, A. Santamarina, G. Noguere, Sensitivity and Uncertainty Analysis applied to the JHR Reactivity Prediction, in Proc. of the PHYSOR 2012: Advances in Reactor Physics - Linking Research, Industry and Education (American Nuclear Society, Knoxville, USA, 2012), 2010

[13] N. Soppera, M. Bossant, E. Dupont, Nuclear Data Sheets 120, 294 (2014) 
